\title{
Gut Microbiota and the Pathogenesis of Insulin Resistance
}

\author{
Nathalie M. Delzenne • Patrice D. Cani
}

Published online: 24 March 2011

(C) Springer Science+Business Media, LLC 2011

\begin{abstract}
Several reviews recently explored how the gut microbiota was able to control host energy metabolism, and thereby the development of adiposity. In this review, we focused on the state of the art that supports a link between the gut microbiota composition and activity, and the management of glycemia associated with overweight and diabetes. Several microbial-derived compounds are related to disturbances of glucose homeostasis including the gramnegative-derived lipopolysaccharides. Some nutrients with prebiotic properties, which escape the digestion in the upper part of the gut, modify the composition of the gut microbiota in favor of bacteria that could play a beneficial role on glucose homeostasis, namely by modulating the endocrine function of the gut, and by reinforcing the gut barrier. Adequate intervention studies in diabetic patients are required to assess the relevance of those experimental data for human health.
\end{abstract}

Keywords Gut microbiota - Diabetes · Prebiotics .

Inflammation $\cdot$ Gut $\cdot$ Lipopolysaccharides

\section{Introduction}

The gut microbiota, composed of hundreds of billions of bacteria, plays an important role in maintaining key

N. M. Delzenne $\cdot$ P. D. Cani

Louvain Drug Research Institute, Metabolism and Nutrition

Research Group, Université Catholique de Louvain,

Brussels, Belgium

N. M. Delzenne $(\triangle)$

LDRI/MNUT, UCL,

Av. E. Mounier, 73,

1200 Brussels, Belgium

e-mail: nathalie.delzenne@uclouvain.be physiologic functions for the "host," by interacting with nondigestible food components, on the one hand, and with human cells, not only in the intestine itself, but also at a distance [1-3•]. Interestingly, DNA fingerprinting approaches performed in mice revealed that the genetic background-more than the gender itself-impacts the composition of the gut microbiota [4]. Experimental data collected in several recent reviews explored how the gut microbiota was able to control host energy metabolism, and thereby the development of adiposity [5-7]. In the studies published initially by Backhed et al. [7, 8], germ-free mice, compared with conventionally raised mice (bearing the gut microbiota), are protected against high-fat diet-induced obesity, but also against associated metabolic disorders, including glucose intolerance [9]. Rabot et al. [10] recently confirmed that the germ-free mice excrete more lipids, consume less calories, and have a lower body weight than the conventional animals. They also show enhanced insulin sensitivity, reduced fasting and nonfasting insulinemia, and increased phospho-Akt in adipose tissue, which are all signs of an improvement of glucose homeostasis. Apparently, the "resistance" of the germ-free mice toward a highfat diet does not occur in all cases, and is dependent on the composition of the diet, thereby suggesting that it is rather the interaction between the gut microbes and the nutrients than the presence of the gut microbiota, that could drive the development of obesity and related diseases [11].

Intervention studies in germ-free mice also support the idea that changes in gut microbiota may be involved in the pathogenesis of the obese and diabetic phenotype. For example, transfer of gut microbes isolated from toll-like receptor (TLR) 5-deficient mice (mice lacking an important component of the innate immune system that develop the features of the metabolic syndrome) to wild-type germ-free animals allow the transfer of the phenotype of the TLR5deficient mice, including insulin resistance, the later effect 
being independent on any effect on food intake [12••]. Such data support the view that the gut microbiota, which composition is different in obese versus lean individuals, is an actor to consider in the management of fat mass development and obesity-related disorders, including diabetes. There are several open questions that will be discussed in the different sections of this paper. First, which components released by the gut microbiota could play a harmful or a beneficial role in the control of glycemia? Second, are there some bacteria that are positively or negatively associated with the occurrence of diabetes, and, if it is the case, how could they play a role in the development of the disease? Third, is it possible to suggest that the gut microbiota can be a target for the development of new drugs or in the evolution of nutritional advices in the control of diabetes and insulin resistance?

\section{The Role of the Microbial-derived Compounds in the Control of Glucose Homeostasis}

The impact of high-fat diet on metabolic disorders linked to gut microbiota proposes that some bacterial-related components (lipopolysaccharides [LPS] - the main component of the gram-negative bacterial membrane-) and TLRs are potential drivers of the occurrence of hyperglycemia and insulin resistance. An increase in LPS concentrations occurs in obese and diabetic animals, but also in humans [13]. In fact, the endotoxemia remains quite modest (the concentration of LPS is approximately doubled in obesity), but when reproduced by chronic injection of exogenous LPS, is able to reproduce the loss of insulin sensitivity present in the obese mice [14, 15]. Moreover, the CD14 knockout and TLR4 knockout animals are resistant to the high-fat diet-induced insulin resistance $[15,16]$. Interestingly, a similar increase in LPS is observed in diabetic patients, the level of LPS being significantly associated with fasting insulin [17••], [18]. There is also an association between the serum level of LPS-binding protein and the metabolic syndrome, including fasting plasma glucose [19].

The endotoxemia induced by high-fat diet feeding is not likely to be due to any increase in gram-negative bacteria [20], but might be linked to an increase in fat-induced LPS absorption, to an increase in gut permeability, or to a decrease in LPS catabolism. Endotoxemia correlates with fat intake in healthy men, thereby contributing to the increase in serum proinflammatory cytokines [21-23]. Alterations of the gut permeability occur in obese and diabetic mice, by a process involving dysfunctions of the tight junction proteins, and the activation of the intestinal endocannabinoid system $[24,25 \bullet]$. The increase in the gut barrier permeability, shown in animals with an oral test with fluorescent-labeled dextran, significantly correlates with the level of LPS in the blood of obese and diabetic animals [24]. Diet-induced obesity-prone, but not diet-induced obesity-resistant, rats exhibit an increase in TLR4 activation associated with a decrease in intestinal alkaline phosphatase, thereby suggesting that a default in the process of LPS degradation by the intestinal alkaline phosphatase would also contribute to the endotoxemia associated with the metabolic disorders associated with obesity [26]. From those data-mostly issued from experimental work performed in animals, and therefore needing to be confirmed in humans - it seems relevant to think that the increase in circulating LPS-a "harmful" component of the gut microbiota-could be part of the inflammatory stress occurring upon obesity and diabetes, and that all the processes participating to the endotoxemia could constitute novel targets in the development of drug and nutrients in the management of the metabolic syndrome.

The gut microbiota can also modulate the glycemia by other processes. Some experimental data, comparing the metabolic behavior of germ-free and conventional animals, suggest that the gut microbiota could help the host to improve the digestion/absorption of ingested nutrients, namely by promoting intestinal monosaccharides absorption [7]. This could contribute to an increase in postprandial glycemia. However, the mechanism of this effect and the relevance in humans remains to be proven. Conversely, by ingesting nutrients that escape the digestion to the upper part of the gut, the host feeds gut microbes, which are able to ferment food components into short-chain fatty acids (SCFAs; acetate propionate, butyrate), which are absorbed in the lower gut, thereby harvesting energy. However, this process of "energy harvest" represents a minor part of potential energy recuperation in (over)fed animals.

Also, these SCFAs clearly contribute to the regulation of host energy metabolism. SCFAs are able to act as signaling molecules in host tissues, by linking selected G-proteincoupled receptors, GPR41 (also called free fatty acid receptor FFAR3) and GPR43 (FFAR2) [27]. Samuel et al. [28] demonstrated that GPR41-/- mice colonized with fermentative microbes (B. thetaiotaiomicron and M. smithii) did not gain fat mass at the same extent as wild-type littermates did. Other data have shown that SFCAs (acetate, propionate) may stimulate adipogenesis via GPR43 activation [29]. These results suggest that the SCFAs could play a harmful role in the context of obesity and related diseases. However, there are several arguments to propose that their production is rather helpful in the control of obesity and related diseases [30॰]. For example, propionate has been shown to inhibit de novo fatty acid synthesis, and, even if it is a gluconeogenic precursor, it inhibits the hepatic gluconeogenesis from lactate in hepatocytes. It has also been shown to improve insulin sensitivity, to induce satiety, 
and to exert anti-inflammatory effects [30•]. Conversely, the administration of sodium butyrate is able to prevent or treat high-fat diet-induced insulin resistance in mice, by promoting energy expenditure and by regulating mitochondrial function in the skeletal muscle and in brown fat [31]. Finally, nondigestible carbohydrates, which are largely fermented in the colon, and which therefore produce large amounts of SCFAs, have been proposed to reduce glycemia and other disorders associated with obesity [32].

\section{Changes in Microbiota Composition Related to Diabetes and Insulin Resistance in Obese or Overweight Individuals}

At this stage, one might think that the gut microbiota could play a harmful role in the context of obesity, because as a whole (conventional vs germ-free mice) it contributes to promote fat mass development. However, both observational and interventional studies in animals and in humans suggest that qualitative changes of the gut microbiota occur upon obesity, thereby wondering about the more "harmful" and more "beneficial" bacteria in this context. Eighty percent to $90 \%$ of the bacterial phylotypes are members of two phyla: the Bacteroidetes (eg, Bacteroides, Prevotella) and the Firmicutes (eg, Clostridium, Enterococcus, Lactobacillus, Ruminococcus), followed by the Actinobacteria (eg, Bifidobacterium) and the Proteobacteria (eg, Helicobacter, Escherichia).

The first original studies describing qualitative changes of the gut microbiota in obese individuals were published by Ley et al. [33] a few years ago. The same author has recently reviewed the data reporting the changes in the composition of the gut microbiota in obese versus lean individuals [5]. Changes in the proportion of the phyla (Bacteroidetes/Firmicutes) occur, but the decrease in this ratio, even if it was initially pointed out, is not necessarily confirmed in all studies [34, 35]. Studies performed in RELM $\beta$ knockout mice, which are resistant to fat-induced obesity, suggest that fat diet itself, and not the obese state, may account for the changes in microbiota composition [36]. Similar conclusions were issued from data relating the changes in the microbial composition and the energy harvesting capacity in $o b / o b$ mice and high-fat diet-fed mice [37]. In addition, specific bacterial gender and species are related to body weight and fat mass. Staphylococcus aureus counting was lower in children who maintain a normal weight than in children becoming overweight several years later. The authors proposed that $S$. aureus may act as a trigger of low-grade inflammation [38], which contributes to the development of obesity and associated metabolic diseases. In agreement with these last findings, Collado et al. [39] found, by analyzing body weight evolution in pregnant women, significantly higher numbers of Bacteroides group and of $S$. aureus in overweight compared with normal weight women. In animals, dietinduced and genetic obesity are characterized by changes in gut microbiota toward a decreased number of bifidobacteria [20]. Those bacteria have been shown to improve the mucosal barrier function, and thereby could play an antiinflammatory role [40]. The Bifidobacterium group is more represented in normal weight than in overweight women and also in women with lower weight gain over pregnancy [39]. Other studies also confirmed that the number of bifidobacteria is lower in obese than in lean individuals [41]. The composition of the gut microbiota in maternal colostrum, or in measured babies, suggest bifidobacteria count might be considered as an index of maintenance of healthy body weight [42••], [43]. Recent reports suggest that the level of bifidobacteria is dependent on the dietary intake because weight loss after bariatric surgery is associated with reduced Bifidobacterium [44・•], whereas decreased Bifidobacterium bifidum and Bifidobacterium breve counts and increased Bifidobacterium catenulatum are observed upon weight loss upon dietary intervention in adolescents [45].

The relationship between the gut microbiota composition and diabetes has been analyzed from several angles. Larsen et al. [46••] analyzed, by microsequencing of the V4 region of the 16SrRNA gene, the gut microbiota composition of 36 adult males including 18 diabetic subjects. The proportion of the phylum Firmicutes was significantly reduced in the diabetic groups versus controls, and that the ratio of several phyla and/or groups (ratio Bacteroidetes/Firmicutes phyla and ratio Bacteroides-Prevotella/C. coccoides-E. rectale groups) correlated positively with fasting glycemia independent on any relationship with the body mass index [46••]. Wu et al. [47••] performed a molecular analysis (polymerase chain reaction and denaturating gel electrophoresis analysis of the $16 \mathrm{~S}$ rRNA genes) of the bacteria in the feces of 16 type 2 diabetic patients and 12 healthy individuals. They showed that the bacterial composition of diabetic group was different from that of the healthy group, the genus Bifidobacterium and Bacteroides vulgatus being less represented in the microbiota of the diabetic than nondiabetic group. The analysis of the diversity profile also suggests that the changes in the gut microbiota can be due to the diabetes status [47••]. Furet et al. [44.•] analyzed the gut microbiota composition in 13 lean and 30 obese individuals including seven type 2 diabetic patients, the later being subjected to a Roux-en-Y gastric bypass. They found, interestingly, that Faecalibacterium prausnitzii species-which has been inversely related previously with inflammatory bowel disease and infection colitis - could also play a role in the low-grade inflammation associated with obesity. They observed that 
the proportion of $F$. prausnitzii was directly linked to the reduction of low-grade inflammation upon intervention in type 2 diabetic subjects, independently of calorie intake, and that there was a negative correlation between $F$. prausnitzii and insulin resistance (homeostasis model assessment). Those data indicate $F$. prausnitzii as a potential microbial target that could help control glucose homeostasis in the context of type 2 diabetes and obesity. The metabolomic analysis also shows the relationship between the gut microbiota and the diabetic status. Individuals with impaired glucose tolerance exhibit a decrease in serum and urinary level of gut microbiota-related metabolites [48].

\section{The Gut Microbiota: A Novel Target in the Nutritional Control of Diabetes and Insulin Resistance}

Several nondigestible/fermentable oligosaccharides exhibit prebiotic properties, namely because they change the composition and/or the activity of the gut microbiota and thereby exert beneficial effects for the host [49]. In obese animals, the dietary supplementation with inulin-type fructans boosts the number of bifidobacteria in the cecum, as well as the production of hormones secreted by host gut endocrine $\mathrm{L}$ cells, including peptide $\mathrm{YY}$ and glucagon-like peptide (GLP-1 and GLP-2) [13, 24, 50, 51]. The production of GLP-1 is namely responsible for the improvement of hepatic insulin resistance and steatosis, for the decrease in blood glucose, and for the maintenance of satiety level in obese mice or rats [50]. Recent data suggest that GPR43 (FFAR2) activation by the SCFAs released upon fructans fermentation could participate in the higher GLP-1 release in the colon of rats treated with fructo-oligosaccharides for 4 weeks [52]. The increase in GLP-2, together with the modulation of the endocannabinoid response in the gut, participates in the restoration of the gut barrier function and thereby lessens endotoxemia $[24,25 \cdot]$. Interestingly, prebiotics treatment is able to decrease interleukin-18 and interleukin-1 $\beta$ serum level in genetic obese mice $(o b / o b)$ and in high-fat-fed mice [24], those two cytokines being considered as gut microbialrelated immunologic factors that drive obesity $[12 \bullet \bullet]$.

The modulation of gut peptides by fructans-type prebiotics has been shown in intervention studies in healthy individuals. An increase in postprandial GLP-1, peptide YY, and gastric inhibitory peptide correlates with a decreased glycemic response, and a decrease in energy intake in healthy individuals supplemented with inulin-type fructans for 2 weeks [51]. A reduced ghrelin level and an increased plasma GLP-1 occurs after a single dose of inulin given in a high fructose corn syrup [53]. Treating obese or diabetic individuals with prebiotics has been tried in a limited number of studies. A partial body weight loss was observed in intervention studies with prebiotics in overweight or obese individuals. Interestingly, some of them showed a decrease in postprandial glycemia and a modulation of the serum level of gut peptides controlling appetite [54-56].

Only a few papers have been published that report the influence of the prebiotic approach on glucose homeostasis in diabetic individuals. In type 2 diabetic patients, no significant modification of glucose homeostasis (plasma glucose level, hepatic glucose production) occurred in prebiotics-treated patients [57]. In a similar study conducted in hypercholesterolemic patients, prebiotics (inulin-type fructans) treatment reduced the postprandial insulin response, but the clinical relevance of this effect remained unclear [58]. Interestingly, some data suggest that wheat fiber intake promotes the colonic fermentation and increases mean plasma GLP-1 in hyperinsulinemic patients, but the observation of this effect requires several months [59]. There is a crucial need for intervention studies that would relate the changes in the gut microbiota composition with the potential improvement of the metabolic disorders in a key target population, namely in diabetic or glucoseintolerant patients.

\section{Conclusion and Perspectives}

Experimental data in animals, but also the analysis of the gut microbiota composition, reveal that specific changes in bacteria (decrease in bifidobacteria, decrease in F. prausnitzii, increase in $S$. aureus) could be linked to the occurrence of inflammation and diabetes in obese humans. The gut microbiota is so complex that it is unrealistic to point out a specific type of bacteria that would be exclusively associated with type 2 diabetes. However, nutritional modulation of the gut microbiota leads to changes in endogenous host targets, which have been considered important for drug development in controlling insulin resistance. For example, food components with prebiotic properties are able to promote the endogenous release of peptides, which are nowadays molecules of drug development for controlling obesity and diabetes, such as glucagon-like peptides. Therefore, the consideration of the gut microbiota as a novel partner in the control of metabolic disorders including insulin resistance requires basic research devoted to elucidate the mediators of the host microbe's dialogue. Adequate nutritional/pharmacologic intervention studies dedicated to addressing the link between modulation of the gut microbiota composition or activity and health improvement in glucose-intolerant or diabetic patients are needed to point out the key (metabolic functions of) gut microbes to promote in the management of obesity and diabetes. 
Disclosure Conflicts of interest: N.M. Delzenne: is a recipient of subsides from the Fonds National de la Recherche Scientifique (FNRS/FRSM) and from the "fonds spéciaux de recherche," UCL (Université catholique de Louvain); P.D. Cani: is research associate from the FRS-FNRS (Fonds de la Recherche Scientifique, Belgique), and is a recipient of subsides from the Fonds National de la Recherche Scientifique (FNRS/FRSM) and from the "fonds spéciaux de recherche," UCL (Université catholique de Louvain).

\section{References}

Papers of particular interest, published recently, have been highlighted as:

- Of importance

•- Of major importance

1. Cani PD, Delzenne NM: The gut microbiome as therapeutic target. Pharmacol Ther 2011. doi:10.1016/J.PHARMTHERA.2011.01.012

2. Jia W, Li H, Zhao L, Nicholson JK. Gut microbiota: a potential new territory for drug targeting. Nat Rev Drug Discov. 2008;7:123-9.

3. - Backhed F, Crawford PA. Coordinated regulation of the metabolome and lipidome at the host-microbial interface. Biochim Biophys Acta. 2010;1801:240-5. This paper reviews the collected data related to the metabolomic approach to evaluate microbialhost interactions.

4. Kovacs A, Ben-Jacob N, Tayem H, et al. Genotype is a stronger determinant than sex of the mouse gut microbiota. Microb Ecol. 2011;61:423-8.

5. Ley RE. Obesity and the human microbiome. Curr Opin Gastroenterol. 2010;26:5-11.

6. Cani PD, Delzenne NM. Interplay between obesity and associated metabolic disorders: new insights into the gut microbiota. Curr Opin Pharmacol. 2009;9:737-43.

7. Backhed F, Ding $\mathrm{H}$, Wang $\mathrm{T}$, et al. The gut microbiota as an environmental factor that regulates fat storage. Proc Natl Acad Sci USA. 2004;101:15718-23.

8. Backhed F, Manchester JK, Semenkovich CF, Gordon JI. Mechanisms underlying the resistance to diet-induced obesity in germ-free mice 1. Proc Natl Acad Sci USA. 2007;104:979-84.

9. Diamant M, Blaak EE, de Vos WM: Do nutrient-gut-microbiota interactions play a role in human obesity, insulin resistance and type 2 diabetes? Obes Rev 2010.

10. Rabot S, Membrez M, Bruneau A, et al. Germ-free C57BL/6 J mice are resistant to high-fat-diet-induced insulin resistance and have altered cholesterol metabolism. FASEB J. 2010;24:4948-59.

11. Fleissner CK, Huebel N, Abd El-Bary MM, et al. Absence of intestinal microbiota does not protect mice from diet-induced obesity. Br J Nutr. 2010;104:919-29.

12. • Vijay-Kumar M, Aitken JD, Carvalho FA, et al. Metabolic syndrome and altered gut microbiota in mice lacking Toll-like receptor 5. Science. 2010;328:228-31. This is one of the key papers showing the transferability of the diabetic phenotype by inoculation of the gut microbiota to germ-free mice.

13. Delzenne NM, Cani PD. Nutritional modulation of gut microbiota in the context of obesity and insulin resistance: Potential interest of prebiotics. Int Dairy J. 2010;20:277-80.

14. Cani PD, Bibiloni R, Knauf C, et al. Changes in gut microbiota control metabolic endotoxemia-induced inflammation in high-fat diet-induced obesity and diabetes in mice. Diabetes. 2008;57:1470 81
15. Cani PD, Amar J, Iglesias MA, et al. Metabolic endotoxemia initiates obesity and insulin resistance. Diabetes. 2007;56:176172.

16. Shi H, Kokoeva MV, Inouye K, et al. TLR4 links innate immunity and fatty acid-induced insulin resistance. J Clin Invest. 2006;116:3015-25.

17. •• Basu S, Haghiac M, Surace P, et al. Pregravid obesity associates with increased maternal endotoxemia and metabolic inflammation. Obesity (Silver Spring). 2011;19:476-82. This is interesting data relating the inflammation status and microbial changes upon pregnancy and fat mass development.

18. Creely SJ, McTernan PG, Kusminski CM, et al. Lipopolysaccharide activates an innate immune system response in human adipose tissue in obesity and type 2 diabetes. Am J Physiol Endocrinol Metab. 2007;292:E740-7.

19. Sun L, Yu Z, Ye X, et al. A marker of endotoxemia is associated with obesity and related metabolic disorders in apparently healthy Chinese. Diab Care. 2010;33:1925-32.

20. Cani PD, Neyrinck AM, Fava F, et al. Selective increases of bifidobacteria in gut microflora improve high-fat-diet-induced diabetes in mice through a mechanism associated with endotoxaemia. Diabetologia. 2007;50:2374-83.

21. Amar J, Burcelin R, Ruidavets JB, et al. Energy intake is associated with endotoxemia in apparently healthy men. Am J Clin Nutr. 2008;87:1219-23.

22. Ghanim H, Abuaysheh S, Sia CL, et al. Increase in plasma endotoxin concentrations and the expression of Toll-like receptors and suppressor of cytokine signaling-3 in mononuclear cells after a high-fat, high-carbohydrate meal: implications for insulin resistance. Diab Care. 2009;32:2281-7.

23. Erridge C, Attina T, Spickett CM, Webb DJ. A high-fat meal induces low-grade endotoxemia: evidence of a novel mechanism of postprandial inflammation. Am J Clin Nutr. 2007;86:1286-92.

24. Cani PD, Possemiers S, Van de WT, et al. Changes in gut microbiota control inflammation in obese mice through a mechanism involving GLP-2-driven improvement of gut permeability. Gut. 2009;58:1091-103.

25. - Muccioli GG, Naslain D, Backhed F, et al. The endocannabinoid system links gut microbiota to adipogenesis. Mol Syst Biol. 2010;6:392. This is a paper describing the implication of the endocannabinoid system in the intestine and in the adipose tissue in the host response toward gut microbiota.

26. de La Serre CB, Ellis CL, Lee J, et al. Propensity to high-fat dietinduced obesity in rats is associated with changes in the gut microbiota and gut inflammation. Am J Physiol Gastrointest Liver Physiol. 2010;299:G440-8.

27. Le PE, Loison C, Struyf S, et al. Functional characterization of human receptors for short chain fatty acids and their role in polymorphonuclear cell activation 1 . J Biol Chem. 2003;278:25481-9.

28. Samuel BS, Shaito A, Motoike T, et al. Effects of the gut microbiota on host adiposity are modulated by the short-chain fatty-acid binding $\mathrm{G}$ protein-coupled receptor, Gpr41. Proc Natl Acad Sci USA. 2008;105:16767-72.

29. Hong YH, Nishimura Y, Hishikawa D, et al. Acetate and propionate short chain fatty acids stimulate adipogenesis via GPCR43. Endocrinology. 2005;146:5092-9.

30. - Al-Lahham SH, Peppelenbosch MP, Roelofsen H, et al. Biological effects of propionic acid in humans; metabolism, potential applications and underlying mechanisms. Biochim Biophys Acta. 2010;1801:1175-83. This is a complete review of the state of the art concerning the metabolic regulation by propionate.

31. Gao Z, Yin J, Zhang J, et al. Butyrate improves insulin sensitivity and increases energy expenditure in mice. Diabetes. 2009;58:150917. 
32. Neyrinck AM, Delzenne NM. Potential interest of gut microbial changes induced by non-digestible carbohydrates of wheat in the management of obesity and related disorders. Curr Opin Clin Nutr Metab Care. 2010;13:722-8.

33. Ley RE, Backhed F, Turnbaugh P, et al. Obesity alters gut microbial ecology. Proc Natl Acad Sci USA. 2005;102:11070-5.

34. Duncan SH, Lobley GE, Holtrop G, et al. Human colonic microbiota associated with diet, obesity and weight loss. Int $\mathrm{J}$ Obes (Lond). 2008;32:1720-4.

35. Zhang H, DiBaise JK, Zuccolo A, et al. Human gut microbiota in obesity and after gastric bypass. Proc Natl Acad Sci USA. 2009;106:2365-70.

36. Hildebrandt MA, Hoffmann C, Sherrill-Mix SA, et al. High-fat diet determines the composition of the murine gut microbiome independently of obesity. Gastroenterology. 2009;137:1716-24.

37. Murphy EF, Cotter PD, Healy S, et al. Composition and energy harvesting capacity of the gut microbiota: relationship to diet, obesity and time in mouse models. Gut. 2010;59:1635-42.

38. Kalliomaki M, Collado MC, Salminen S, Isolauri E. Early differences in fecal microbiota composition in children may predict overweight. Am J Clin Nutr. 2008;87:534-8.

39. Collado MC, Isolauri E, Laitinen K, Salminen S. Distinct composition of gut microbiota during pregnancy in overweight and normal-weight women. 1. Am J Clin Nutr. 2008;88:894-9.

40. O'Mahony D, Murphy S, Boileau T, et al. Bifidobacterium animalis AHC7 protects against pathogen-induced NF-kappaB activation in vivo. BMC Immunol. 2010;11:63.

41. Schwiertz A, Taras D, Schafer K, et al. Microbiota and SCFA in lean and overweight healthy subjects. Obes Silver Spring. 2010;18:190-5.

42. •- Luoto R, Kalliomaki M, Laitinen K, et al. Initial dietary and microbiological environments deviate in normal-weight compared to overweight children at 10 years of age. J Pediatr Gastroenterol Nutr. 2011;52:90-5. This is interesting data supporting the relevance of the changes in microbiota early in life on the occurrence of overweight and obesity.

43. Luoto R, Laitinen K, Nermes M, Isolauri E. Impact of maternal probiotic-supplemented dietary counselling on pregnancy outcome and prenatal and postnatal growth: a double-blind, placebocontrolled study. Br J Nutr. 2010;103:1792-9.

44. •- Furet JP, Kong LC, Tap J, et al. Differential adaptation of human gut microbiota to bariatric surgery-induced weight loss: links with metabolic and low-grade inflammation markers. Diabetes. 2010;59:3049-57. This is a key paper describing the relationship between the level of F. Prausnitzii in the fecal samples of obese humans, and the development of diabetes and inflammation.

45. Santacruz A, Collado MC, Garcia-Valdes L, et al. Gut microbiota composition is associated with body weight, weight gain and biochemical parameters in pregnant women. $\mathrm{Br} \mathrm{J}$ Nutr. 2010;104:83-92.

46. •- Larsen N, Vogensen FK, van den Berg FW, et al. Gut microbiota in human adults with type 2 diabetes differs from non-diabetic adults. PLoS One. 2010;5:e9085. This is novel data comparing the changes in gut in the profile of bacteria in diabetic and nondiabetic patients.

47. $\bullet \mathrm{Wu} \mathrm{X}, \mathrm{Ma} \mathrm{C}$, Han L, et al. Molecular characterisation of the faecal microbiota in patients with type II diabetes. Curr Microbiol. 2010;61:69-78. This is one of the rare data published in humans that report the changes in gut microbiota with a focus on bifidobacteria composition in diabetic patients.

48. Zhao X, Fritsche J, Wang J, et al. Metabonomic fingerprints of fasting plasma and spot urine reveal human pre-diabetic metabolic traits. Metabolomics. 2010;6:362-74.

49. Roberfroid M, Gibson GR, Hoyles L, et al. Prebiotic effects: metabolic and health benefits. Br J Nutr. 2010;104 Supp1 2:S1-63.

50. Cani PD, Knauf C, Iglesias MA, et al. Improvement of glucose tolerance and hepatic insulin sensitivity by oligofructose requires a functional glucagon-like Peptide 1 receptor. Diabetes. 2006;55:1484-90.

51. Cani PD, Lecourt E, Dewulf EM, et al. Gut microbiota fermentation of prebiotics increases satietogenic and incretin gut peptide production with consequences for appetite sensation and glucose response after a meal. Am J Clin Nutr. 2009;90:1236-43.

52. Kaji I, Karaki SI, Tanaka R, Kuwahara A: Density distribution of free fatty acid receptor 2 (FFA2)-expressing and GLP-1-producing enteroendocrine $\mathrm{L}$ cells in human and rat lower intestine, and increased cell numbers after ingestion of fructo-oligosaccharide. $\mathrm{J}$ Mol Histol 2010.

53. Tarini J, Wolever TM. The fermentable fibre inulin increases postprandial serum short-chain fatty acids and reduces free-fatty acids and ghrelin in healthy subjects. Appl Physiol Nutr Metab. 2010;35:9-16.

54. Abrams SA, Griffin IJ, Hawthorne KM, Ellis KJ. Effect of prebiotic supplementation and calcium intake on body mass index. J Pediatr. 2007;151:293-8.

55. Genta S, Cabrera W, Habib N, et al. Yacon syrup: beneficial effects on obesity and insulin resistance in humans. Clin Nutr. 2009;28:182-7.

56. Parnell JA, Reimer RA. Weight loss during oligofructose supplementation is associated with decreased ghrelin and increased peptide YY in overweight and obese adults. Am J Clin Nutr. 2009;89:1751-9.

57. Luo J, Van YM, Rizkalla SW, et al. Chronic consumption of shortchain fructooligosaccharides does not affect basal hepatic glucose production or insulin resistance in type 2 diabetics. J Nutr. 2000;130:1572-7.

58. Giacco R, Clemente G, Luongo D, et al. Effects of short-chain fructo-oligosaccharides on glucose and lipid metabolism in mild hypercholesterolaemic individuals. Clin Nutr. 2004;23:331-40.

59. Freeland KR, Wilson C, Wolever TM. Adaptation of colonic fermentation and glucagon-like peptide- 1 secretion with increased wheat fibre intake for 1 year in hyperinsulinaemic human subjects. Br J Nutr. 2010;103:82-90. 